\title{
BINORMAL COOLING ERRORS IN SINGLE HOT-WIRE MEASUREMENTS
}

\author{
Ramis Örlü, P. Henrik Alfredsson \\ Linné FLOW Centre, KTH Mechanics, Stockholm, Sweden \\ e-mail:ramis@mech.kth.se
}

\begin{abstract}
In single-wire hot-wire measurements, velocity fluctuations acting normal to the hot-wire and its prongs will cause additional heat transfer known as binormal cooling. With respect to wall turbulence, the influence of this additional cooling is well-studied for crossed wires, while it is commonly ignored in single hot-wire measurements. The latter view is challenged in the recent work by Dróżdż and Elsner (2014) that claims significant errors in variance measurements when using single-wire probes in turbulent boundary layers. This short communication revisits these claims and quantifies binormal cooling errors through an expansion of the effective-velocity concept and utilisation of direct numerical simulation data. Results support the common habit that binormal cooling errors can safely be ignored in single hot-wire measurements.
\end{abstract}

Keywords: hot-wire anemometry, measurement errors, wall turbulence

\section{Introduction and motivation}

Hot-wire anemometry is still the method that provides the highest degree of accuracy when measuring turbulent fluctuations, in particular, when it comes to temporal and spatial resolution. Recent detailed comparisons between hot-wire measurements and direct numerical simulations (DNS) in turbulent boundary layer (TBL) flows revealed that most of the remaining differences between hot-wire measurements and DNS can be explained by insufficient spatial resolution of the measuring sensor (Örlü and Schlatter, 2013); but is with sufficient care within the scatter of various DNS. On the other hand, there are still open questions when it comes to the scaling of the streamwise velocity variance profile, in particular, with respect to turbulent pipe flows (Örlü and Alfredsson, 2012). Some of the discrepancies, besides spatial resolution (Hutchins et al., 2009), could recently also be related to end-conduction effects (Miller et al., 2014) and frequency response (Hutchins et al., 2015) effects, while the effect of temperature fluctuations were found to be comparably mild (Örlü et al., 2014).

In a recent study by Dróżdż and Elsner (2014), the streamwise velocity variance profiles obtained from a single-wire (SW) and crossed-wire, i.e. X-wire (XW), probe in a TBL were compared, and the authors concluded that binormal cooling effects (i.e. velocity fluctuations acting normal to the hot-wire and its prongs that cause additional heat transfer) were not negligible. In particular, they claimed "that the underestimation of the near-wall peak of streamwise fluctuating component in X-wire measurements results from disregarded wall-normal fluctuations, which is obviously taken [into account] in the case of a single-wire probe". This statement implies also that all previous comparisons of streamwise velocity statistics between SW probes and other measurement techniques such as laser Doppler velocimetry (LDV) and Particle Image Velocimetry (PIV), but also numerical simulations such as DNS, have apparently compared different quantities. The authors furthermore concluded that the readings from a SW probe should be compared to the sum of energies of the streamwise $(u)$ and wall-normal $(v)$ component from an 
XW probe (assuming the wire is normal to the mean flow direction and parallel to the wall), i.e. $\overline{u u}_{\mathrm{SW}}^{+}=\overline{u u}_{\mathrm{XW}}^{+}+\overline{v v}_{\mathrm{XW}}^{+}$, where the superscript + denotes scaling with wall units and the overbar the time-average operator. These results have consequently been used by the authors in followup studies (Dróżdż and Elsner, 2015) as well as to compare SW results with PIV measurements (Dróżdż and Uruba, 2014).

In absence of a quantification of binormal cooling errors in SW measurements in the literature and in light of the consequences, which the aforementioned claims bring for past and future SW measurements, there is a need to address this problem. This short communication will therefore revisit the statements of Dróżdż and Elsner (2014), present clarifications for their observations as well as quantify binormal cooling errors based on recent DNS data. It is believed that these will not only be useful to remedy the claims made, but also give confidence in past and future SW measurements, which - none-withstanding the progress in optical measurement techniques - remains the measurement technique of choice when single-point streamwise velocity statistics are of interest.

\section{Comments and Results}

Let us start with the relevant statements by Dróżdż and Elsner (2014), which will be reproduced (in italic) and commented on:

1) "Most researchers who do measurements in the turbulent boundary layer believe that the influence of $v$ component is insignificant and can be ignored, ...". Indeed, most researchers employing SW probes in wall-bounded flows ignore the effect of the wall-normal and spanwise velocity component as evident from a large number of studies. On the other hand, the effect of the binormal velocity component in turbulence measurements using XW probes has been studied to some extent. It is e.g. well-known that large errors can be obtained in jet flows (Ovink et al., 2001), while the errors in wall-bounded flows are small, but not negligibly (Zhao and Smits, 2006). Hence, this statement by Dróżdż and Elsner (2014) is correct, i.e. most researchers who do measurements with SW probes "believe" (or know) that the influence of the binormal component is insignificant, while researchers using XW probes are aware of them.

2) "... but it is only a simplifying assumption" and "... from the physical point of view, the negligible small influence of the $v$ component in a single-wire readings is not so convincing." To address this claim, we start out by considering the effective cooling velocity. Accounting for pitch and yaw angles of the effective cooling velocity with respect to a SW aligned normal to the flow and parallel to the wall, the effective cooling velocity $\left(U_{e}\right)$ in a three-dimensional flow is given by (Jørgensen, 1971)

$$
U_{e}^{2}=U^{2}+h^{2} V^{2}+k^{2} W^{2}
$$

where $W$ denotes the spanwise velocity (i.e. parallel to the hot-wire), $k$ the yaw factor which accounts for the effects of finite wire length and the prong orientation with respect to the flow, and $h$ the pitch factor, which is related to the binormal component. There is a rich literature with regard to the values of these two factors, but they are commonly $k \simeq 0.10-0.20$ and $h \simeq 1.02-1.05$ for standard wire probes, while they asymptote to 0 and 1 for an infinitely large length-to-diameter ratio (Bruun, 1995). Since $\bar{W}=0$ and $\bar{V} \approx 0$ (where the overbar indicates the time-average) in the aforementioned canonical wall-bounded flows, $W=w$ and $V \approx v$. Since $h^{2} \gg k^{2}$ and $h \approx 1$, the series expansion of Eq. (2.1) on the assumption that

$$
\left|\frac{u}{U}\right|,\left|\frac{v}{U}\right|,\left|\frac{w}{U}\right| \ll 1
$$


yields for the mean effective cooling velocity

$$
\overline{U_{e}}=\bar{U}\left(1+\frac{v^{\prime 2}}{2 \bar{U}^{2}}+O\left[\frac{u}{U}\right]^{3}\right)
$$

while the measured variance becomes

$$
u_{e}^{\prime 2}=u^{\prime 2}\left(1+\frac{\overline{u v^{2}}}{\bar{U} u^{\prime 2}}-\frac{\overline{u^{2} v^{2}}}{\bar{U}^{2}{u^{\prime}}^{2}}+\frac{\overline{v^{4}}-v^{\prime 4}}{4 \bar{U}^{2} u^{\prime 2}}+O\left[\frac{u}{U}\right]^{5}\right)
$$

where the prime denotes the root mean square (rms) value. Similar expressions for the mean and (a truncated form of the) variance can be found in Bruun (1995). As apparent $\overline{U_{e}} \geqslant \bar{U}$, since the second term in brackets in Eq. (2.3) is per definition positive, while the situation for $u_{e}^{\prime 2}$ is dependent on the sign of the leading order term (i.e. $\sim \overline{u v^{2}}$ ). To assess the error between the measured (effective cooling) velocity and the actual horizontal velocity component, the error for the mean

$$
\varepsilon_{\overline{U_{e}}}=\frac{\overline{U_{e}}-\bar{U}}{\bar{U}}=\frac{v^{\prime 2}}{2 \bar{U}^{2}}
$$

and variance

$$
\varepsilon_{u_{e}^{\prime 2}}=\frac{u_{e}^{\prime 2}-u^{\prime 2}}{u^{\prime 2}}=\frac{\overline{u v^{2}}}{\bar{U} u^{\prime 2}}-\frac{\overline{u^{2} v^{2}}}{\bar{U}^{2} u^{\prime 2}}+\frac{\overline{v^{4}}-v^{\prime}}{4 \bar{U}^{2} u^{\prime 2}}
$$

are obtained.

To demonstrate the effect of the binormal velocity component on the readings of a SW probe aligned normal to the mean flow, and not be affected by data that suffers from insufficient spatial and temporal resolution, here DNS data from a TBL (Schlatter and Örlü, 2010) has been utilized. The response of a SW has been imitated by computing the statistics upon utilization of Eq. (2.1) on the time-series data, cf. Segalini et al. (2011) and Örlü and Schlatter (2013). Figure 1a depicts the "true" and "measured" (i.e. effecttive) mean and variance profile. As apparent, the difference between $\overline{U_{e}}$ and $\bar{U}$ as well as $u_{e}^{\prime 2}$ and $u^{\prime 2}$ is barely visible and can hence, as commonly done, safely be neglected. The obtained statistics can now be used to compute the aforementioned errors and compare them with the derived simplified expressions given above as depicted in Fig. 1b,c. The mean streamwise velocity is overestimated up to $0.3 \%$, while the variance is underestimated
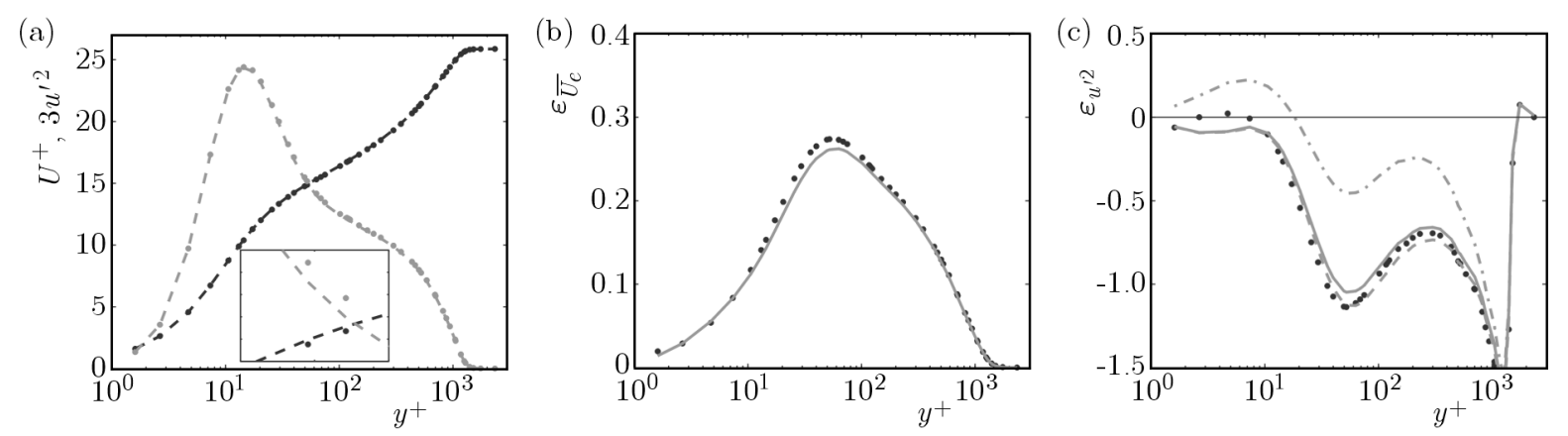

Fig. 1. (a) Inner-scaled mean and variance profile for a TBL at $R e_{\tau} \approx 1200$ (Schlatter and Örlü, 2010) with the dashed line and dots indicating "measured" (i.e. effective) and real values, respectively. Inset shows the region around $y^{+}=50$, where the error in the mean and variance has its maximum.

Percentile error in the (b) mean velocity and (c) variance, where dots indicate the results computed from the DNS through Eq. (2.1) and the solid line represent the approximation given through Eqs. (2.5) and (2.6). The dashed and dash-dotted line in (c) denote Eq. (2.6) with only the first two and the first term, respectively 
up to $1 \%$. This is in contrast to the errors induced in XW measurements when ignoring the binormal velocity, which are approximately fivefold (Zhao and Smits, 2006). Although these errors are obtained for a TBL at a specific Reynolds number, the estimated errors can directly be transferred to pipe and channel flows as well. Furthermore, the errors are representative for a wide range of Reynolds numbers due to the logarithmic dependence of the variance amplitudes (Alfredsson et al., 2011). With regards to the initial statement that "the negligible small influence of the $v$ component in a single-wire readings is not so convincing", it can now clearly be stated that binormal cooling effects on SW probes can safely be neglected in wall-bounded flows, under the premise that assumption (2.2) is not severely violated. ${ }^{1}$

3) Dróżdż and Elsner (2014) furthermore observed, with respect to a XW, that "the vector summing these two components (i.e. $\overline{u u}_{\mathrm{XW}}^{+}$and $\overline{v v}_{\mathrm{XW}}^{+}$), obtained from the X-wire probe, gives the shape of fluctuation distribution obtained from the $S W$ (i.e. $\overline{u u}_{\mathrm{SW}}^{+}$) probe". Consequently, the authors compared $\left(\overline{u u}^{+}+\overline{v v}^{+}\right)_{\mathrm{XW}}$ (Dróżdż and Elsner, 2014) or $\left(\overline{u u}^{+}+\overline{v v}^{+}\right)_{\mathrm{PIV}}$ (Dróżdż and Uruba, 2014) and not directly the measured $\overline{u u^{+}}$component with the variance read from a SW probe and found a seemingly better agreement as demonstrated in Fig. 2a, which is a reproduction from Dróżdż and Elsner (2014). This proposed workaround is, however, at odds with the aforementioned results, which demonstrated that binormal cooling errors are safely negligible in turbulent boundary layer measurements. Streamwise and wall-normal velocity fluctuations are furthermore strongly anti-correlated and the simple addition of the energies is principally only permissible for fully uncorrelated signals. The reason why the summation of measured variances from an XW probe or PIV measurements lead to a better agreement with SW measurements in the work by Dróżdż and Elsner (2014) and Dróżdż and Uruba (2014) is simply related to the larger viscous-scaled wire length utilized for the XW measurements (the length of the XW was around three times longer than that of the SW), which causes attenuation of the fluctuation amplitudes (Örlü and Alfredsson, 2010). This can simply be shown by utilising any of the available spatial resolution correction schemes for hot-wire measurements available in the literature (e.g. Segalini et al., 2011; Smits et al., 2011). As demonstrated in Fig. 2b, the streamwise variance profile measured by the $\mathrm{SW}, \overline{u u}_{\mathrm{SW}}^{+}$, compares well with DNS data and is apparently well-resolved. Matching now the viscous-scaled wire length of the SW with that of the employed XW, by utilization of the scheme by Smits et al. (2011), the variance profile attenuates towards the variance read by the XW, $\overline{u u}_{\mathrm{XW}}^{+}$. The comparison with the DNS data also reveals that the wall-normal variance profile becomes increasingly overestimated the closer

(a)

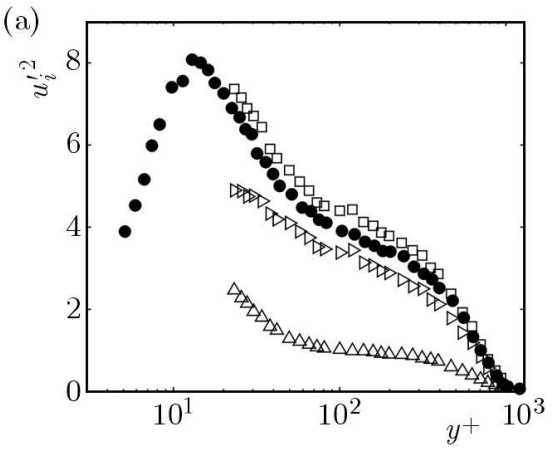

(b)

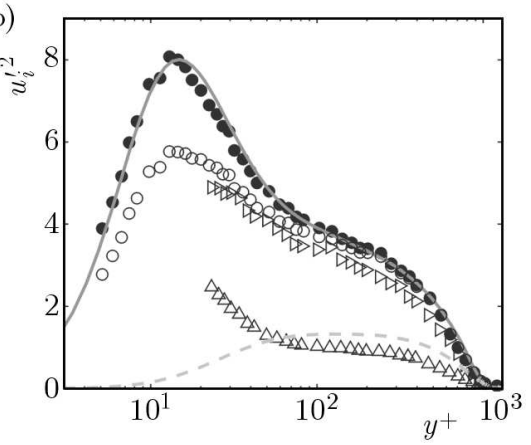

Fig. 2. Inner-scaled variance profile for a TBL at $\operatorname{Re}_{\tau} \approx 1000$ with data taken from Fig. 3 of Dróżdż and Elsner (2014). (a) SW, $\overline{u u}_{\mathrm{SW}}^{+}: \bullet, \mathrm{X}$-wire, $\overline{u u}_{\mathrm{XW}}^{+}: \triangleright, \overline{v v}_{\mathrm{XW}}^{+}: \triangle$, and $\overline{u u}_{\mathrm{XW}}^{+}+\overline{v v}_{\mathrm{XW}}^{+}: \square$; (b) reverse application of the spatial resolution correction scheme by Smits et al. $(2011)$ on the SW data $(\bullet)$ to match the less resolved resolution of the X-wire (o). For reference, also DNS from Schlatter and Örlü (2010) at the same Re for $\overline{u u}{ }^{+}(-)$and $\overline{v v^{+}}(--)$is shown

\footnotetext{
${ }^{1}$ In this respect, it is also worth referring to Kalpakli Vester et al. (2015), where the results from a $\mathrm{SW}$ and PIV measurements in a rotating pipe flow are compared.
} 
to the wall the probe is. Such an increase is well documented and known to be related to the spatial resolution as well as the spacing between the two inclined wires (Talamelli et al., 2000).

\section{Conclusions}

The present short communication addresses the claims made by Dróżdż and Elsner (2014), viz. that binormal cooling errors in SW measurements are not negligible in wall turbulence. They further claimed that the measured variance by a SW probe needs to be compared to the sum of energies from the streamwise and wall-normal components, e.g. when comparing with results from XW or PIV measurements. These claims have been addressed by means of a simple expansion of the effective velocity, which showed that the effect of binormal cooling in SW measurements can - under the premise that assumption (2.2) is not severely violated safely be neglected. The results have also been validated by means of DNS data and provide a quantification of binormal cooling errors, which has been missing in the literature, and might have given rise to the claims by Dróżdż and Elsner (2014).

\section{References}

1. Alfredsson P.H., Segalini A., Örlü R., 2011, A new scaling for the streamwise turbulence intensity in wall-bounded turbulent flows and what it tells us about the "outer" peak, Physics of Fluids, 23, 041702

2. Bruun H.H., 1995, Hot-Wire Anemometry: Principles and Signal Analysis, Oxford University Press Inc., New York, USA

3. DróżDż A., Elsner W., 2014, Comparison of single and X-wire measurements of streamwise velocity fluctuations in turbulent boundary layer, Journal of Theoretical and Applied Mechanics, 52, 499-505

4. DróżDż A., ElSNER W., 2015, Analysis of hot-wire measurements accuracy in turbulent boundary, Open English, 5, 307-313

5. DróżDż A., Uruba V., 2014, Comparison of PIV and hot-wire statistics of turbulent boundary layer, Journal of Physics: Conference Series, 530, 012044

6. Hutchins N., Monty J.P., Hultmark M., Smits A.J., 2015, A direct measure of the frequency response of hot-wire anemometers: temporal resolution issues in wall-bounded turbulence, Experiments in Fluids, 56, 18

7. Hutchins N., Nickels T.B., Marusic I., Chong M.S., 2009, Hot-wire spatial resolution issues in wall-bounded turbulence, Journal of Fluid Mechanics, 635, 103-136

8. Jørgensen F.E., 1971, Directional sensitivity of wire and fiber-film probes, DISA Information, 11, 31-37

9. Kalpakli Vester A., Sattarzadeh S.S., ÖRlü R., 2015, Combined hot-wire and PIV measurements of a swirling turbulent flow at the exit of a $90^{\circ}$ pipe bend, Journal of Visualization, doi:10.1007/s12650-015-0310-1

10. Miller M.A., Estejab B., Bailey S.C.C., 2014, Evaluation of hot-wire spatial filtering corrections for wall turbulence and correction for end-conduction effects, Experiments in Fluids, 55, 1735

11. Örlü R., Alfredsson P.H., 2010, On spatial resolution issues related to time-averaged quantities using hot-wire anemometry, Experiments in Fluids, 49, 101-110

12. Örlü R., Alfredsson P.H., 2012, Comment on the scaling of the near-wall streamwise variance peak in turbulent pipe flows, Experiments in Fluids, 54, 1431 
13. Örlü R., Malizia F., Cimarelli A., Schlatter P., Talamelli A., 2014, The influence of temperature fluctuations on hot-wire measurements in wall-bounded turbulence, Experiments in Fluids, 55, 1781

14. Örlü R., Schlatter P., 2013, Comparison of experiments and simulations for zero pressure gradient turbulent boundary layers at moderate Reynolds numbers, Experiments in Fluids, 54, 1547

15. Ovink R., Lamers A.P.G.G., van Steenhoven A.A., Hoeijmakers H.W.M., 2001, A method of correction for the binormal velocity fluctuation using the look-up inversion method for hot-wire anemometry, Measurement Science and Technology, 12, 1208

16. Schlatter P., Örlü R., 2010, Assessment of direct numerical simulation data of turbulent boundary layers, Journal of Fluid Mechanics, 659, 116-126

17. Segalini A., Örlü R., Schlatter P., Alfredsson P.H., Rüedi J.D., Talamelli A., 2011, A method to estimate turbulence intensity and transverse Taylor microscale in turbulent flows from spatially averaged hot-wire data, Experiments in Fluids, 51, 693-700

18. Smits A.J., Monty J.P., Hultmark M., Bailey S.C.C., Hutchins N., Marusic I., 2011, Spatial resolution correction for wall-bounded turbulence measurements, Journal of Fluid Mechanics, 676, 41-53

19. Talamelli A., Westin K.J.A., Alfredsson P.H., 2000, An experimental investigation of the response of hot-wire X-probes in shear flows, Experiments in Fluids, 28, 425-435

20. Zhao R., Smits A.J., 2006, Binormal cooling errors in crossed hot-wire measurements, Experiments in Fluids, 40, 212-217 